\section{Using the multidimensional prognostic index for treatment decisions and monitoring in frail older patients}

\author{
Arduino A. Mangoni \\ Department of Clinical Pharmacology, \\ College of Medicine and Public Health, \\ Flinders University and Flinders \\ Medical Centre, Adelaide, Australia
}

\begin{abstract}
The routine applicability of clinical guidelines and disease-specific end-points in frail older patients is problematic because of the exclusion of this group from clinical trials, their limited life expectancy, the co-existence of multiple disease states and poor functional status, and the presence of complex drug-drug and drug-disease interactions. In this context, the use of patient-centred end-points that include measures of quality of life might be particularly useful for designing tailored treatment strategies, monitor progress and, ultimately, improve outcomes. The multidimensional prognostic index, an objective, quantifiable, and validated scoring system based on core domains of the comprehensive geriatric assessment, might represent an important tool for the development of clinical guidelines that take into account measures of frailty and patientcentred end-points. However, research is warranted to investigate whether this approach leads to more effective and safe management strategies in old age.
\end{abstract}

\section{Multidimensional prognostic index in frail older patients}

Evidence based medicine has revolutionized the approach of health care practitioners towards diagnosis and treatment of disease over the last 20 years. Professional recommendations for the management of common disease states are regularly updated, both at national and international level, as a result of the thorough assessment of new evidence from the published literature. While the use of clinical guidelines has undoubtedly led to a more consistent and rational approach to patient care the general nature of the recommendations provided fails, by definition, to account for specific patient characteristics. By and large, clinical guidelines target medical conditions that typically affect the older population. However, they generally focus on individual disease states, rarely taking into account the impact of co-morbidities, polypharmacy, drug-drug and disease-drug interactions, and the inter-individual variability in homeostatic capacity on diagnosis, management, and clinical outcomes in old age. Furthermore, the use of disease-specific end-points that are based on objective parameters and biomarkers assessed in traditional clinical trials might be problematic in frail older patients with significant disability and limited life expectancy. ${ }^{1} \mathrm{~A}$ further factor limiting the applicability of clinical guidelines to the routine care of older adults is that this patient group, particularly frail older patients, remains excluded from participating in clinical trials, an essential step in the assessment of the efficacy and safety of treatments. ${ }^{2}$ This is particularly concerning because frail people represent a significant proportion of the older population requiring care in different countries and health care settings. ${ }^{3}$ For these reasons, there is a lack of evidence regarding the safety and efficacy of treatment options in frail older patients that have complex co-morbid states and specific social circumstances. However, unlike current guidelines, such evidence should also be based on the assessment of the impact of disease states on key patient-centred end-points such as measures of functional status and quality of life, as well as life expectancy. In this context, it is important to emphasise that different disease states might similarly affect a patient's quality of life and the capacity to perform daily activities, and that the co-existence of two or more conditions in the same patient might have negative synergic effects on these measures. ${ }^{4}$

A robust assessment of pre-defined patient-centred end-points, however, can only be accomplished using tools that are validated in clinical practice. The comprehensive geriatric assessment (CGA), cornerstone of geriatric care and routinely used by health care staff to assess functional dependence and frailty in hospital and community-dwelling patients, contains information that can be used to characterize patientcentred end-points, such as functional capacity, risk of falls, cognition and behaviour, social support, treatment goals and advance care preferences. ${ }^{5}$ However, an important limitation of the CGA is the lack of objective and quantifiable information that can be used to predict clinical outcomes, including mortality. A quantification of the information provided by the CGA would facilitate the development and validation of safe and effective patient-centred management pathways in frail older patients. A number of tools have been developed, apart from the CGA, to assess frailty and functional status in older
Correspondence:Arduino A. Mangoni, Department of Clinical Pharmacology, College of Medicine and Public Health, Flinders University and Flinders Medical Centre, Bedford Park, SA 5042, Australia. Tel.: +61.8.8204.7495;

Fax: +61.8 .8204 .5114$

E-mail: arduino.mangoni@flinders.edu.au

Key words: Multidimensional prognostic index; comprehensive geriatric assessment; end-points; clinical guidelines; frailty.

Conference presentation: paper presented at $31^{\circ}$ Congresso Nazionale SIGOT - Genova, June 8-9, 2017.

Received for publication: 10 September 2017. Accepted for publication: 14 September 2017.

This work is licensed under a Creative Commons Attribution-NonCommercial 4.0 International License (CC BY-NC 4.0).

(C) Copyright A.A. Mangoni, 2017

Licensee PAGEPress, Italy

Geriatric Care 2017; 3:7059

doi:10.4081/gc.2017.7059

patients. ${ }^{6}$ However, their clinical use in complex real-life geriatric patients is uncertain. ${ }^{7}$ The multidimensional prognostic index (MPI), developed by Pilotto et al., ${ }^{8}$ provides a quantifiable score that is based on eight CGA domains: i) activities of daily living; ii) instrumental activities of daily living; iii) short portable mental status questionnaire; iv) mini-nutritional assessment; v) Exton-Smith scale; vi) cumulative index rating scale; vii) total number of medications; and viii) social support network

The MPI has shown superior predictive capacity and discrimination in relation to a number of adverse outcomes in observational studies of older populations, particularly hospital length of stay, readmission rates, and mortality, when compared to other tools. ${ }^{9}$ Furthermore, initial evidence suggests that the MPI can be considered as universal patient-centred end-point to assess the effects of pharmacological and non-pharmacological interventions in patients with dementia and depression. ${ }^{10,11}$ This approach allows the quantification of the effects of therapy on the MPI score as a whole as well as on the scores of individual components of the tool.

Therefore, the available evidence suggests that tools such as the MPI might provide objective, quantitative, data on key baseline patient characteristics such as functional status, burden of disease, polypharmacy, and life expectancy. This information might be used for designing clinical guide- 
lines and tailored treatments in frail older patients and, in some cases, for the early identification of treatment futility. At the same time, treatment-induced changes in the MPI might also allow to establish the magnitude of the effect on patient-centred endpoint, to further confirm, or refute, the efficacy and safety of therapies in this group.

However, a key step to facilitate the use of the MPI for treatment decisions and patient monitoring in clinical practice remains the inclusion of older patients, particularly those $>80$ years and frail, in clinical trials and the assessment of patient-centred, in addition to disease-centred, end-points in these studies. ${ }^{1,2}$ Only then, can a proper evidence base approach support the routine clinical use of the MPI in patient care.

\section{References}

1. Mangoni AA, Pilotto A. New drugs and patient-centred end-points in old age: setting the wheels in motion. Expert
Rev Clin Pharmacol 2016;9:81-9.

2. Mangoni AA, Jansen PA, Jackson SH. Under-representation of older adults in pharmacokinetic and pharmacodynamic studies: a solvable problem? Expert Rev Clin Pharmacol 2013;6:35-9.

3. Buckinx F, Rolland Y, Reginster JY, et al. Burden of frailty in the elderly population: perspectives for a public health challenge. Arch Public Health 2015; 73:19.

4. Tinetti ME, McAvay GJ, Chang SS, et al. Contribution of multiple chronic conditions to universal health outcomes. J Am Geriatr Soc 2011;59:1686-91.

5. Stuck AE, Siu AL, Wieland GD, et al. Comprehensive geriatric assessment: a meta-analysis of controlled trials. Lancet 1993;342:1032-6.

6. Rodriguez-Manas L, Fried LP. Frailty in the clinical scenario. Lancet 2015;385: e7-9.

7. Deckx L, van den Akker M, Daniels L, et al. Geriatric screening tools are of limited value to predict decline in functional status and quality of life: results of a cohort study. BMC Fam Pract 2015;16:30.
8. Pilotto A, Ferrucci L, Franceschi M, et al. Development and validation of a multidimensional prognostic index for one-year mortality from comprehensive geriatric assessment in hospitalized older patients. Rejuvenation Res 2008; 11:151-61.

9. Pilotto A, Rengo F, Marchionni N, et al. Comparing the prognostic accuracy for all-cause mortality of frailty instruments: a multicentre 1-year follow-up in hospitalized older patients. PLoS One 2012;7:e29090.

10. D'Onofrio G, Sancarlo D, Addante F, et al. A pilot randomized controlled trial evaluating an integrated treatment of rivastigmine transdermal patch and cognitive stimulation in patients with Alzheimer's disease. Int J Geriatr Psychiatry 2015;30:965-75.

11. Pilotto A, D’Onofrio G, Panza F, et al. Treatment of late-life major depressive disorder with selective serotonin reuptake inhibitors improves the multidimensional prognostic index. J Clin Psychopharmacol 2012;32:726-9. 